\title{
Elaboration and Electrochemical Studies of the Coating Behavior of a New Nanofunctional Epoxy Polymer on E24 Steel in $3.5 \% \mathrm{NaCl}$
}

\author{
Rachid Hsissou $^{a, *}$, Bouchra Benzidia ${ }^{b}$, Najat Hajjaji ${ }^{b}$ and Ahmed Elharfi ${ }^{a}$ \\ ${ }^{a}$ Laboratory of Agro-resources, Polymers and Process Engineering (LAPPE), Team of \\ Organic Chemistry and Polymers (TOCP), Department of Chemistry, Faculty of \\ Sciences, \\ Ibn Tofail University, P.O. Box 133, 14000, Kénitra, Morocco \\ ${ }^{b}$ Laboratory of Materials, Electrochemistry and Environment, Team of Corrosion, Protection \\ and Environment, Department of Chemistry, Faculty of Sciences, \\ Ibn Tofail University, P.O. Box 133, 14000, Kénitra, Morocco
}

Received February 13, 2017; accepted, December 2, 2017

\begin{abstract}
The goal of our work is to develop, study, characterize and apply new epoxy macromolecular matrices in the coating process, and to optimize them in the conservation of marine heritage. Epoxy resins are technologically and nanotechnologically compatible thermosetting macromolecule matrices, which are easy to use, thanks to their structures and viscosimetric and rheological properties, able to protect the heritage subject to atmospheric corrosion. In this paper, we tested the synthesized, crosslinked and formulated novel macromolecular nanoglycidyl trihydrazine 4,4,4-tripropoxy ethylene tribisphenol A (NGTHTPETBA), used as an anti-corrosive coating for $\mathrm{E} 24$ steel in $3.5 \% \mathrm{NaCl}$. In order to evaluate the inhibiting performance of the NGTHTPTBAE coating for E24 steel corrosion, and to examine its coating behavior, we applied the different $\mathrm{E}_{1}$ (NGTHTPETBA/MDA) and $\mathrm{E}_{2}$ (NGTHTPETBA/MDA/PN) formulations. The stationary and transient electrochemical studies are very revealing.
\end{abstract}

Keywords: NGTHTPETBA, coating, E24 steel, adsorption phenomenon, crosslinking, formulation and electrochemical studies.

\section{Introduction}

Polyepoxide resins are very important thermosetting technological macromolecular matrices [1-2], which have been widely used in several industrial fields, such as radioactive waste conditioning [3-4], viscosimetric and rheological behavior [5-6-7], aeronautical construction [8], spatial construction [9-10], and metallic objects corrosion inhibition [11] and coating [12]. Our goal

\footnotetext{
* Corresponding author. E-mail address: r.hsissou@gmail.com
} 
is to assess a new behavior of an E24 steel coating, which is easy to implement and inexpensive for the heritage protection subjected to standard atmospheric corrosion. The field of heritage conservation has borrowed some corrosion protection means that were developed for industrial applications, such as the socalled chemical or electrochemical conversion coatings, metallic deposits or organic polymer films, which are now commonly used. As a result, these processes have been used in the field of heritage conservation. This study consists in physico-chemically applying and studying a new polyepoxide resin, and its anticorrosive behavior against E24 steel in marine environments. These materials can be corroded in aggressive media [13-14] and, therefore, the best way to protect them against corrosion is a coating application [15-16]. Among these various types of coatings we have found organic polymers, which play a very important role in preventing the oxidation corrosion, and also avoiding the heritage degradation [17-18]. The coating method with epoxy resins remains a very effective approach to avoid rapid corrosion, because of its good adhesion, good acid/alkali resistance, and its high cross-linking density, thanks to its macromolecular structure [19].

In this work, we applied a new nanofunctional epoxy prepolymer, nanoglycidyl trihydrazine 4,4,4-tripropoxy of ethylene tribisphenol A, synthesized in the laboratory [20], on E24 steel, in marine environments. The coating impact on E24 steel was assessed by using the two $E_{1}$ (NGTHTPETBA/MDA) and $E_{2}$ (NGTHTPETBA/MDA/PN) protection formulations, while evaluating their anticorrosive behavior by two electrochemical methods, namely the stationary method and the transitional method.

\section{Materials and methods \\ Hardware}

\section{Used products}

In this work, we used the nanofunctional epoxide prepolymer nanoglycidyl trihydrazine 4.4.4-tripropoxy of ethylene tribisphenol A (NGTHTPETBA), synthesized in the laboratory [20], methylene dianiline (MDA), natural phosphate and $3.5 \% \mathrm{NaCl}$. The latter has been marketed by Aldrich Chemical Co.

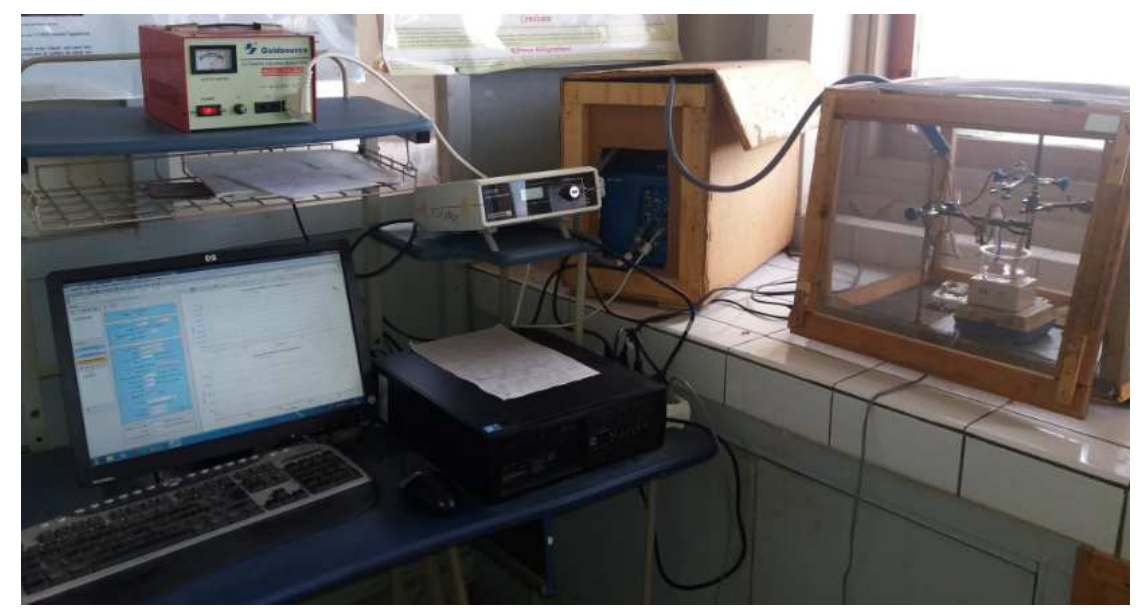

Figure 1. The electrochemical cell with three electrodes. 


\section{Used methods}

Electrochemical cell with three electrodes

Electrochemical measurements were obtained by using an electrochemical cell with three electrodes, as shown in Fig. 1.

There is a platinum electrode as a counter electrode, a saturated calomel reference electrode (ECS) and a cylindrical E24 steel working electrode. The surface of contact with the corrosive solution was $1 \mathrm{~cm}^{2}$. Before each test, the working electrode was polished with abrasive papers: 600, 1200 and 1500.

These three electrodes were immersed in a $100 \mathrm{~mL}$ container, and inserted in its orifices with well-designed diameters and spacings, making it possible to receive the systems for agitation, temperature control, aeration and deaeration.

\section{Electrochemical measurements}

By using a potentiometer/galvanostat SP-200 Biologic Science Instruments, the stationary measurements were carried out in a potentiodynamic mode. The working electrode was previously kept immersed at the free corrosion potential for 30 minutes. The scanning speed was $1 \mathrm{mV} / \mathrm{s}$. The determination of the electrochemical parameters ( $i_{\text {corr }}, E_{\text {corr }}, \beta_{\mathrm{a}}$ and $\beta_{\mathrm{c}}$ ) from the polarization curves was carried out by using a nonlinear regression by the Ec-Lab software. Therefore, the coating efficiency was calculated through the following formula:

$$
\eta \%=\left(\frac{i_{c o r r}^{0}-i_{c o r r}}{i_{c o r r}^{0}}\right) \times 100
$$

where $\mathrm{i}^{\circ}$ corr and $\mathrm{i}_{\text {corr }}$ are the corrosion current densities $\left(\mathrm{A} \cdot \mathrm{cm}^{-2}\right)$, respectively, without and with the coating polymer's different formulations.

$\beta_{\mathrm{a}}$ and $\beta_{\mathrm{c}}$ are the Tafel constants of the anodic and cathodic reactions $\left(\mathrm{V}^{-1}\right)$, respectively. These constants are related to the slope of Tafel $\beta\left(\mathrm{V} \cdot \operatorname{dec}^{-1}\right)$ and the form on the logarithmic scale given by:

$$
\beta=\frac{\ln (10)}{b}=\frac{2.303}{b}
$$

The electrochemical impedance spectroscopy measurements were carried out by using the same apparatus, with a signal amplitude of $10 \mathrm{mV}$. The explored frequency ranged from $100 \mathrm{KHz}$ to $10 \mathrm{MHz}$. The results were then analyzed by using an equivalent electrical circuit of Bouckamp simulation program [19]. The effectiveness of the coating protection was evaluated by using the following relationship:

$$
\eta \%=\left(\frac{R_{p}-R_{p}^{0}}{R_{p}}\right) \times 100
$$

where $\mathrm{R}_{\mathrm{p}}^{\circ}$ and $\mathrm{R}_{\mathrm{p}}$ are the polarization resistance, respectively, in the absence and presence of the coating polymer's different formulations. 
The hardening of nanoglycidyl trihydrazine 4,4,4-tripropoxy of ethylene tribisphenol A (NGTHTPETBA)

The polyepoxide resins can be converted into thermosetting macromolecular matrices, because many of their chemical compounds function as curing agents during the process. Among the hardeners of polyepoxide systems, there are two major classes: acid anhydrides and amines. The latter remain good hardeners thanks to their chemical structure. The aromatic compounds provide very good thermal stability and mechanical properties to the polymer [21]. They are often used for high-tech applications. The hardener which we are using is methylene dianiline, of which chemical structure is shown in Fig. 2:

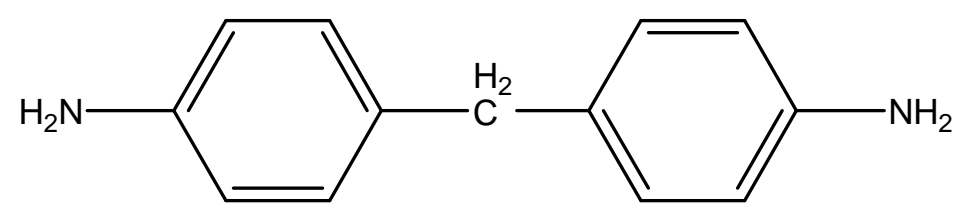

Figure 2. Semi-developed formula of methylene dianiline.

The impact of methylene dianiline on the epoxide is described by the following reaction, according to Fig. 3:
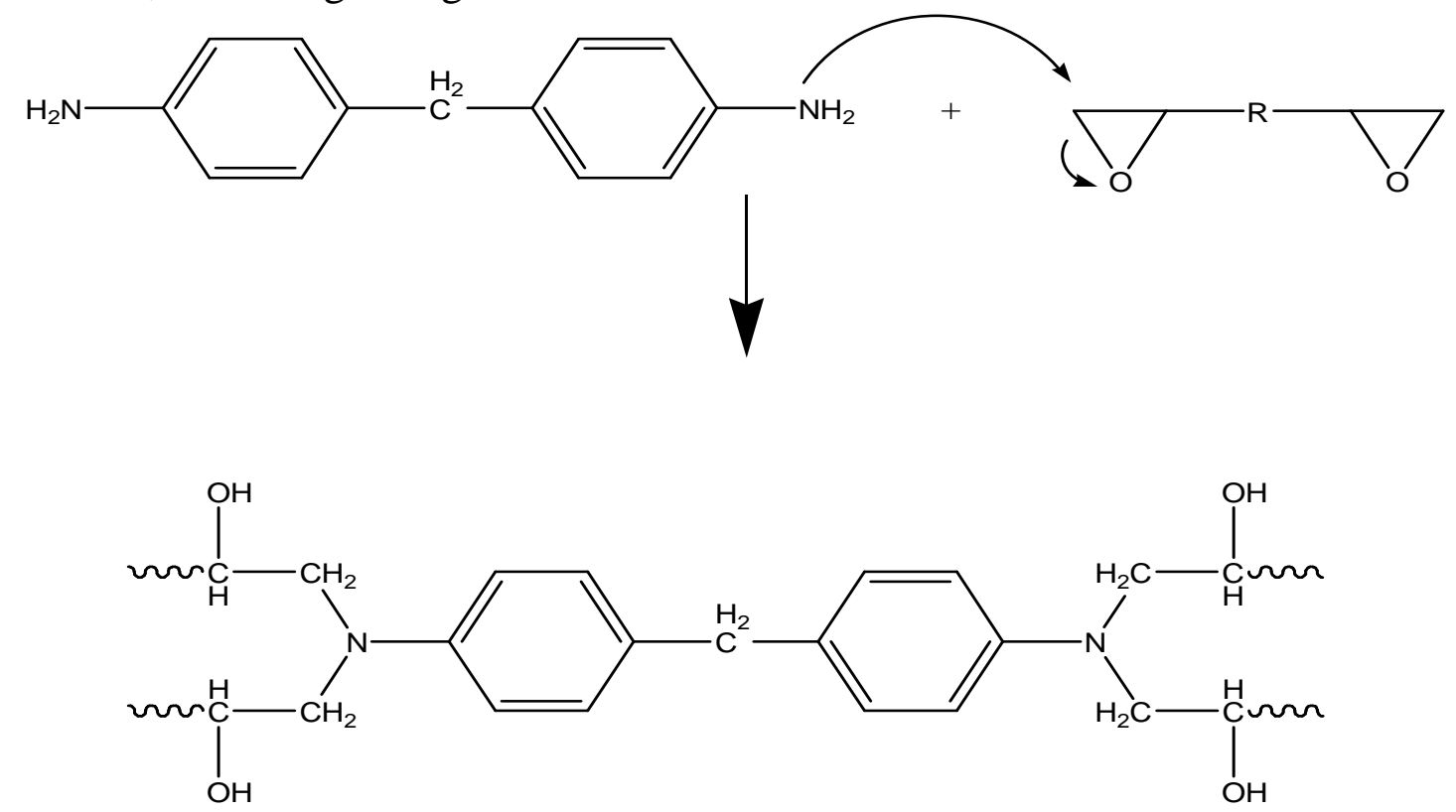

Figure 3. Diagram of NGTHTPETBA resin crosslinked with MDA.

\section{Ratio calculation}

\section{Calculation of stoichiometric coefficients}

Once having cured the multifunctional polyepoxide macromolecular matrix in the hardener presence (especially amines), in order to obtain its optimum properties, it is desirable to make the prepolymer and the curing agent react to approximately stoichiometric amounts [7]. 
Calculation of the epoxy equivalent (EEW) to the synthesized nanoglycidyl trihydrazine 4,4,4-tripropoxy of ethylene tribisphenol A macromolecular matrix is as follows:

$$
E E W=\frac{M_{w}(\text { NGTHTPETBA })}{f}
$$

with $\mathrm{f}$ as the functionality of the synthesized epoxy resin, where:

$$
\begin{gathered}
E E W=\frac{1486}{9} \\
E E W=165 g / e q
\end{gathered}
$$

The calculated AHEW (Amine Hydrogen Equivalent Weight) constitutes the mass of the hardener containing an amine equivalent.

$$
A H E W=\frac{M_{w}(M D A)}{f}
$$

Methylene dianiline: $\mathrm{M}_{\mathrm{w}}=198 ; \mathrm{f}=4$

$$
\begin{aligned}
& A H E W=\frac{198}{4} \\
& A H E W=49.5 \mathrm{~g} / \mathrm{eq}
\end{aligned}
$$

Calculation of the ratio by weight

The hardener-resin ratio by weight was calculated, in the majority of cases, per PHR (parts per hundred of resin):

$$
A \min e(P H R)=\frac{A H E W}{E E W} \times 100
$$

In this case, for NGTHTPETBA:

$$
\begin{aligned}
& A \min e(P H R)=\frac{49.5}{165} \times 100 \\
& A \min e(P H R)=30 g / e q
\end{aligned}
$$

Therefore, $30 \mathrm{~g}$ of methylene dianiline per $100 \mathrm{~g}$ of nanoglycidyl trihydrazine 4,4,4-tripropoxy of ethylene tribisphenol A had to react with the prepolymer, curing it, for it to achieve optimum properties. 
Calculation of the load quantity

We calculated the quantity of the desired load according to the following equation:

$$
y \%=\frac{x}{r e \sin +M D A+x}
$$

E24 steel metal coating by nanoglycidyl trihydrazine 4,4,4-tripropoxy of ethylene tribisphenol $A$

To obtain reliable and reproducible results, the plate was subjected, before each test, to the surface polishing with abrasive papers of increasingly fine grain sizes: 600,1200 and 1500. The used experimental protocol is as follows: Formulation 1 (NGTHTPETBA/MDA) - we mixed $1 \mathrm{~g}$ of NGTHTPETBA with $0.3 \mathrm{~g}$ of MDA, as a hardener, and then stirred it to give a single fluid phase for about $30 \mathrm{~min}$. The formulation was applied to E24 steel by using a film-producing rod. This coated plate was placed in the oven at $70{ }^{\circ} \mathrm{C}$ for 24 hours, to crosslink the polymer deposited onto the substrate; Formulation 2 (NGTHTPETBA/MDA/PN) - we followed the previous protocol (Formulation 1) with the addition of $5 \%$ of natural phosphate as load.

The used electrolyte $(3.5 \% \mathrm{NaCl})$

We used $3.5 \% \mathrm{NaCl}$ as a corrosive solution. We prepared $100 \mathrm{~mL}$ of $3.5 \% \mathrm{NaCl}$, and then we dissolved $3.5 \mathrm{~g}$ of it in $100 \mathrm{~mL}$ of distilled water. The used polyepoxide resin was NGTHTPETBA.

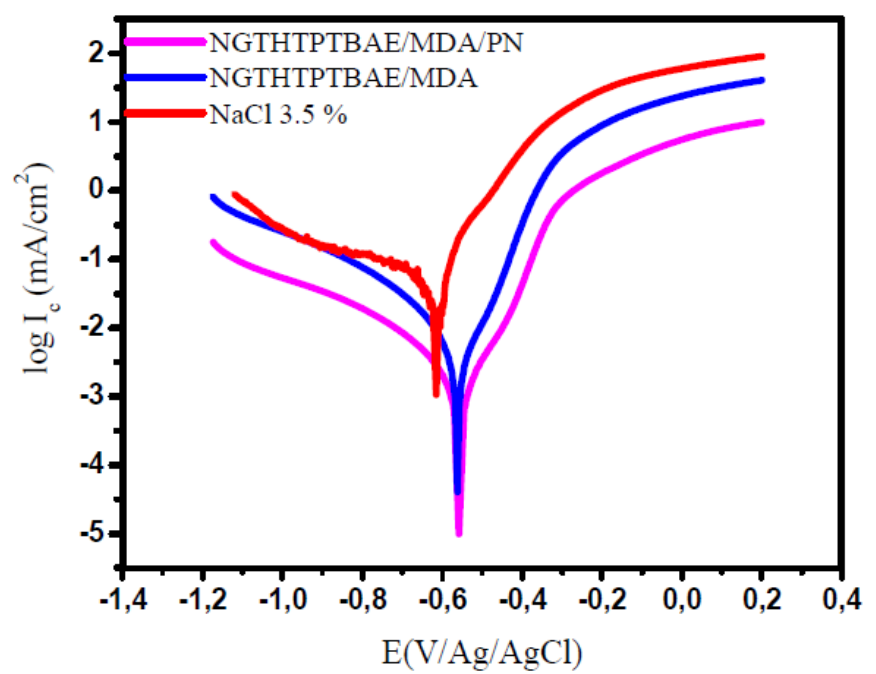

Figure 4. Polarization curves of $\mathrm{E} 24$ steel in $3.5 \% \mathrm{NaCl}$, in the presence of the matrices $\left(\mathrm{E}_{0}, \mathrm{E}_{1}\right.$ and $\left.\mathrm{E}_{2}\right)$, after 30 min of immersion at $298 \mathrm{~K}$.

In Fig. 4, we notice that the cathodic branches do not show linearity, and are considered to be the sum of two curves (showing mixed kinetics) by reducing oxygen and hydrogen [22]. However, the anodic branches also do not show linearity, due to the E24 steel dissolution [23]. 
Table 1. Accumulations of the various electrochemical parameters recorded from the polarization curves.

\begin{tabular}{|l|c|c|c|c|c|c|}
\hline \multicolumn{1}{|c|}{ Protective matrices } & $\begin{array}{c}\text { Plaques } \\
(\mathbf{E i})\end{array}$ & $\begin{array}{c}-\mathbf{E}_{\mathbf{c o r r}} \\
(\mathbf{m V / A g / A g C l})\end{array}$ & $\begin{array}{c}\mathbf{I}_{\mathbf{c o r}} \\
\left(\boldsymbol{\mu} \mathbf{A} / \mathbf{c m}^{2}\right)\end{array}$ & $\begin{array}{c}\beta_{\mathbf{c}} \\
(\mathbf{m V / d e c})\end{array}$ & $\begin{array}{c}\beta_{\mathbf{a}} \\
(\mathbf{m V / d e c})\end{array}$ & $\begin{array}{c}\mathbf{\eta} \\
(\%)\end{array}$ \\
\hline $3.5 \%$ in NaCl & $\mathrm{E}_{0}$ & 665 & 22 & 275 & 78 & - \\
\hline NGTHTPETBA/MDA & $\mathrm{E}_{1}$ & 562 & 1.98 & 82 & 80 & 91 \\
\hline NGTHTPETBA/MDA/PN & $\mathrm{E}_{2}$ & 558 & 0.80 & 110 & 98.5 & 96 \\
\hline
\end{tabular}

In this section, we have studied the protecting mechanism for E24 steel by the matrices $\left(E_{0}, E_{1}\right.$ and $\left.E_{2}\right)$, which consist of the standard polyepoxide resin (NGTHTPETBA), the hardener (MDA) and the natural phosphate load. The results interpretation, according to the matrix constitution, is as follows:

In the control $\mathrm{E}_{0}$ matrix, which was made without the substrate coating, the $\mathrm{Cl}^{-}$ and $\mathrm{OH}^{-}$anions coming from the corrosive medium directly attack the substrate, which induces direct corrosion.

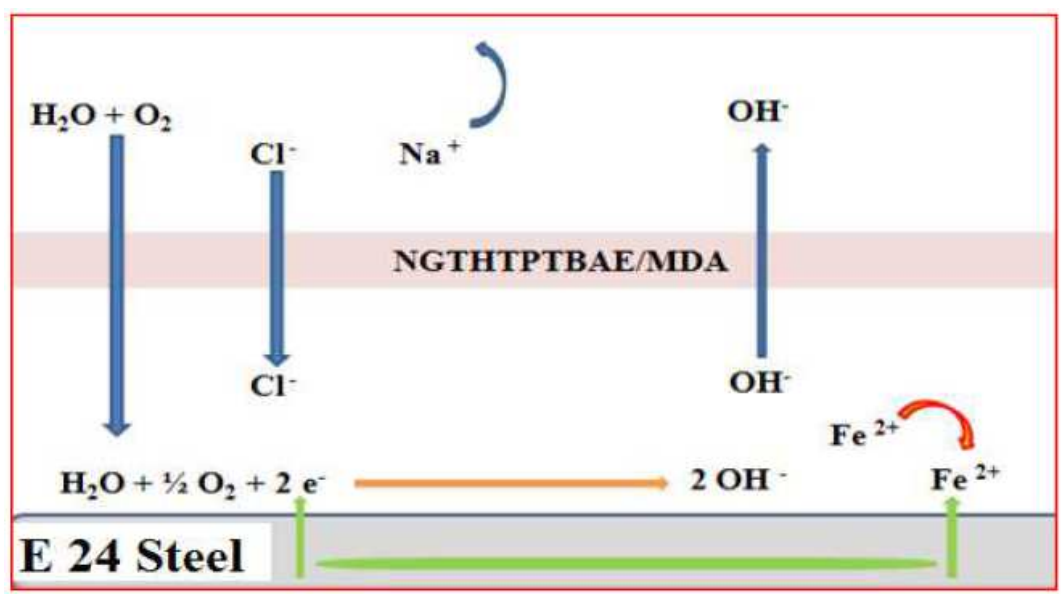

Figure 5. Mechanism of the $E_{1}$ protective matrix (NGTHTPTBAE/MDA) amid the metal substrate and the film, after immersion in $3.5 \% \mathrm{NaCl}$.

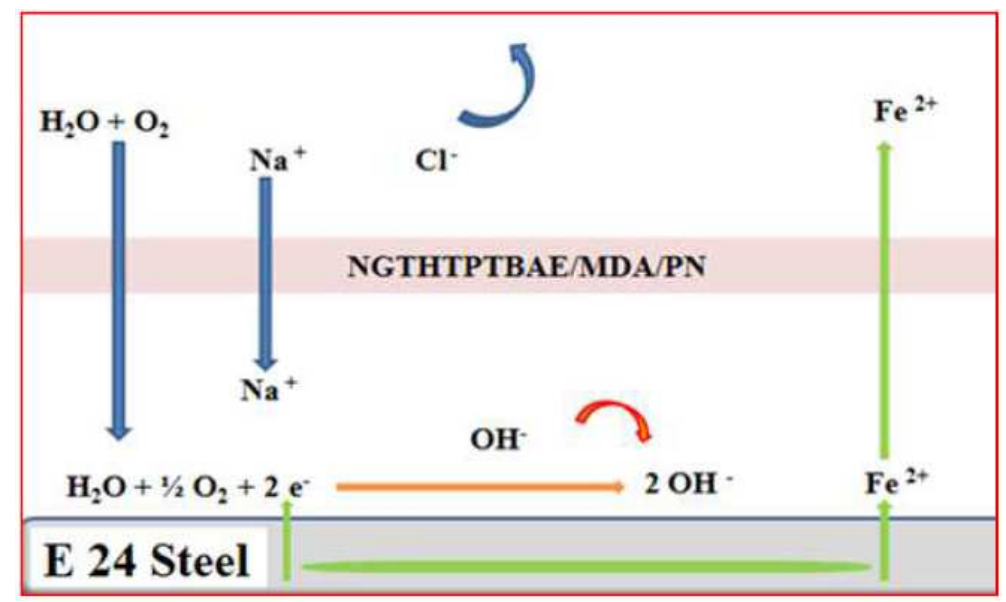

Figure 6. Protective mechanism of the $\mathrm{E}_{2}$ matrix (NGTHTPETBA/MDA/PN) amid the metal substrate and the film, after immersion in $3.5 \% \mathrm{NaCl}$. 
Fig. 5 and 6 show the protection mechanism for E24 steel by the $E_{1}$ (NGTHTPETBA/MDA) and $\mathrm{E}_{2}$ (NGTHTPETBA/MDA/PN) formulations, respectively without and with the natural phosphate.

Thanks to the $\mathrm{E}_{1}$ matrix (NGTHTPETBA/MDA), we anticipate that there is a relatively large diffusion of chloride and hydroxyl ions through the protective film, which could have micropores, by inducing the primary corrosion, translated by $\mathrm{i}_{\text {corr }}=1.98 \mu \mathrm{A} / \mathrm{cm}^{2}$ and $\Pi \%=91 \%$. This would lead to an acceleration of the metal anodic dissolution [24]. The $\mathrm{E}_{1}$ matrix protection mechanism is shown in Fig. 5.

Once having applied $\mathrm{E}_{2}$ matrix (NGTHTPETBA/MDA/PN), we have found that the corrosive species $\left(\mathrm{Cl}^{-}\right.$and $\left.\mathrm{OH}^{-}\right)$diffusion is slowed down by the layer formed by natural phosphate [25-26]. In this case, the electrochemical corrosion parameters are $i_{\text {corr }}=0.80 \mu \mathrm{A} / \mathrm{cm}^{2}$ and $\Pi=96 \%$. The protection mechanism for the $\mathrm{E}_{2}$ matrix is shown in Fig. 6.

The corrosion potential mainly moves towards the anode direction, with $\mathrm{E}_{1}$ (NGTHTPETBA/MDA) and $\mathrm{E}_{2}$ (NGTHTPETBA/MDA/PN) matrices. The addition of $5 \%$ of natural phosphate is accompanied by a marked decrease in corrosion current, in both cathodic and anodic domains. This allowed us to conclude that natural phosphate addition to the formulation made it act as a mixed inhibitor.

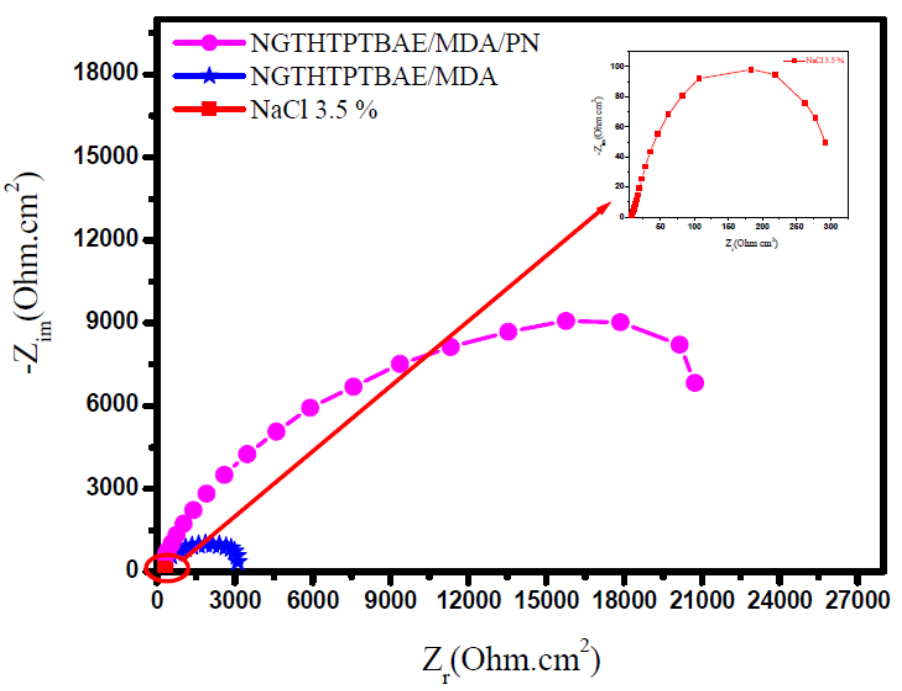

Figure 7. Open-circuit electrochemical impedance diagrams after immersing E24 steel in $3.5 \% \mathrm{NaCl}$, in the matrices $\left(\mathrm{E}_{0}, \mathrm{E}_{1}\right.$ and $\left.\mathrm{E}_{2}\right)$ presence, at $298 \mathrm{~K}$.

\section{Electrochemical impedance spectroscopy}

In order to study in detail the mechanism of coating behavior, we performed electrochemical impedance spectroscopy, of which results are represented in the form of Nyquist diagrams. These impedance diagrams of the E24 steel, immersed 30 min before each open circuit measurement in the corrosive $3.5 \% \mathrm{NaCl}$ solution, with respect to the set of matrices $\left(E_{0}, E_{1}\right.$ and $\left.E_{2}\right)$, are presented in Fig. 7. This confirms the results obtained by the potentiodynamic polarization curves. The analysis of the different diagrams on the composition of the protective matrices $\left(E_{0}, E_{1}\right.$ and $\left.E_{2}\right)$ shows that they are formed by a high-frequency 
capacitive loop attributed to the film effect, which is due to the diffusion phenomenon. In fact, the more the semi-circle diameter increases, the better is the corrosion resistance of the protective film [27]. This study confirms the results obtained by the stationary method.

Furthermore, Fig. 8 and 9, respectively, show the Bode and the phase angle patterns of the E24 steel coating behavior in a marine environment. The plots of these diagrams in the presence of different formulations, $E_{0}, E_{1}$ and $E_{2}$, are illustrated in the figures below.

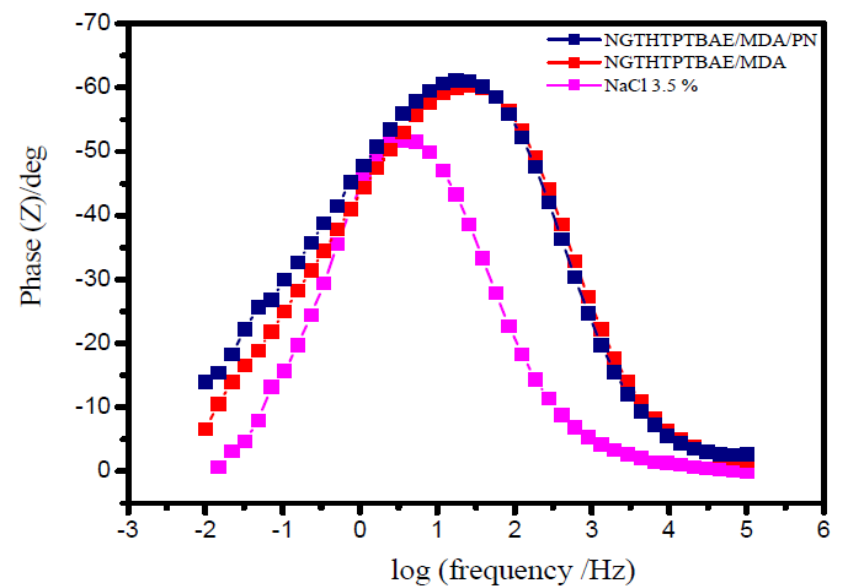

Figure 8. Phase angle diagrams of NGTHTPETBA in $3.5 \% \mathrm{NaCl}$, after $30 \mathrm{~min}$ of immersion in different formulations $\left(\mathrm{E}_{0}, \mathrm{E}_{1}\right.$ and $\left.\mathrm{E}_{2}\right)$ at $298 \mathrm{~K}$.

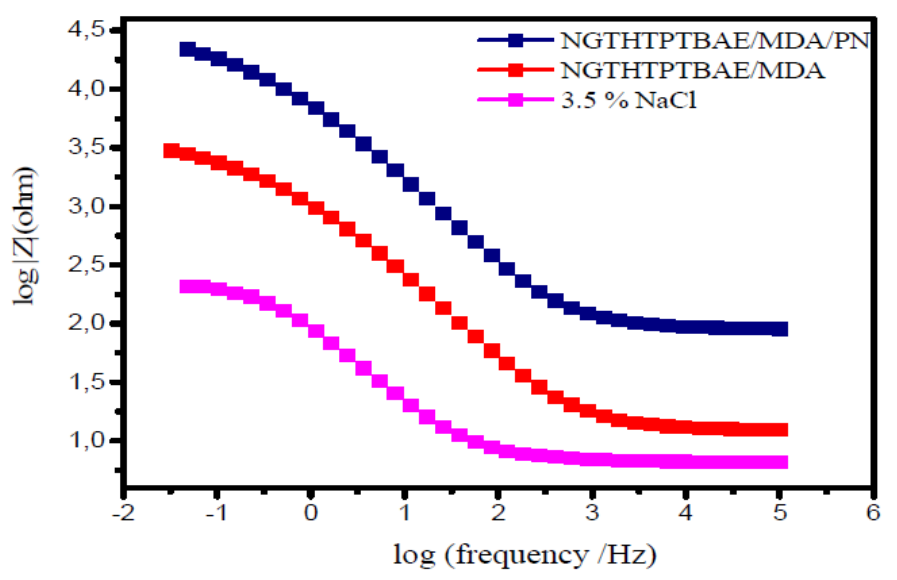

Figure 9. Bode diagrams of NGTHTPETBA in 3.5\% NaCl, after $30 \mathrm{~min}$ of immersion in different formulations $\left(\mathrm{E}_{0}, \mathrm{E}_{1}\right.$ and $\left.\mathrm{E}_{2}\right)$, at $298 \mathrm{~K}$.

According to Fig. 8, we have found that the phase angles increased with the number of coating constituents. This increase in phase angles confirms a higher protection obtained by stronger coating formulations $\left(E_{0}, E_{1}\right.$ and $\left.E_{2}\right)$. Indeed, from this phase angle diagram, we have found that there are three frequency domains: low frequencies, high frequencies and intermediate frequencies. For low frequencies, the increase in the impedance absolute values confirms the greatest protection with different coating formulations for E24 steel. At high frequency, the phase angle values are approximately equal to zero. This indicates that the electrode behavior corresponds to the solution resistance [28]. For the 
intermediate frequencies, the phase angle is close to $70^{\circ}$, and a linear relationship between $\log |\mathrm{Z}|$ in view of $\log$ (f), with a slope close to -1 , was observed. The latter shows the capacitive behavior of the coating at intermediate frequencies.

In the literature, the condenser's behavior would be ideal, if the slope value reached -1 , and if a phase angle value reached $90^{\circ}$. The phase angle values are calculated from the phase curves $(Z)$ in view of $\log$ (frequency). These values of $\alpha$ vary between -0.50 and -0.70 for the different coating formulations, which could be related to the non-ideal structure of the metal/solution interface. These results confirm those of electrochemical impedance spectroscopy.

According to the presented curve shapes, the Bode and Nyquist diagrams show a capacitive behavior at the interface, and the existence of an equivalent electrical circuit (Fig. 10), which is due to the interpretation of the information on the behavior properties of the metal surface coating or corrosion. This circuit is composed of: $\mathrm{R}_{\mathrm{s}}$ (electrolyte resistance); $\mathrm{C}_{\mathrm{f}}$ (coating film capacity); $\mathrm{R}_{\mathrm{p}}$ (coating film resistance); $\mathrm{R}_{\mathrm{ct}}$ (load transfer resistor); and $\mathrm{C}_{\mathrm{dl}}$ (double layer capacity).

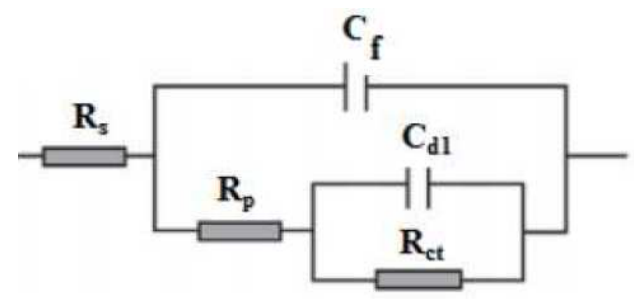

Figure 10. Equivalent electrical circuit of the impedance diagrams obtained in the presence of different formulations for $\mathrm{E} 24$ steel in $3.5 \% \mathrm{NaCl}$.

The electrochemical impedance values and the efficiency resulting from each protection matrix $\left(\mathrm{E}_{0}, \mathrm{E}_{1}\right.$ and $\left.\mathrm{E}_{2}\right)$ are grouped in Table 2.

Table 2: Various electrochemical parameters taken from the impedance diagrams.

\begin{tabular}{|c|c|c|c|c|c|c|}
\hline $\begin{array}{c}\text { Protective } \\
\text { matrices } \mathbf{E}_{\mathbf{i}}\end{array}$ & $\begin{array}{c}\text { Rs } \\
\left(\Omega \cdot \mathbf{c m}^{2}\right)\end{array}$ & $\begin{array}{c}\text { Ret } \\
\left(\Omega . \mathrm{cm}^{2}\right)\end{array}$ & $\begin{array}{c}\mathrm{C}_{\mathrm{dl}} \\
\left(\mu \mathrm{F} / \mathrm{cm}^{2}\right)\end{array}$ & $\begin{array}{c}\mathbf{C}_{\mathbf{f}} \\
\left(\mu \mathrm{F} / \mathbf{c m}^{2}\right)\end{array}$ & $\begin{array}{c}\mathbf{R}_{\mathbf{p}} \\
\left(\Omega . \mathbf{c m}^{2}\right)\end{array}$ & $\underset{(\%)}{\eta}$ \\
\hline $\mathrm{E}_{0}$ & 6.5 & 2.40 & 1253 & 935 & 218.6 & - \\
\hline$E_{1}$ & 12 & 5 & 236 & 11.4 & 3519 & 94 \\
\hline $\mathrm{E}_{2}$ & 84.9 & 31.8 & 34 & 1.94 & 29346 & 99 \\
\hline
\end{tabular}

From this table, we conclude that the polarization resistance values of the coating film for the $E_{0}, E_{1}$ and $E_{2}$ protective matrices confirm the parameters results obtained from the potentiodynamic polarization curves. Table 3 summarizes the results of the stationary and transient methods.

Table 3. Evaluation parameters of the stationary and transient methods.

\begin{tabular}{|c|c|c|c|c|}
\hline \multirow{2}{*}{$\mathbf{E}_{\mathbf{i}}$} & \multicolumn{2}{|c|}{ stationary method } & \multicolumn{2}{c|}{ transient method } \\
\cline { 2 - 5 } & $\begin{array}{c}\mathbf{I}_{\mathbf{c o r r}} \\
\left(\boldsymbol{\mu} \mathbf{A} / \mathbf{c m}^{\mathbf{2}}\right)\end{array}$ & $\begin{array}{c}\boldsymbol{\eta} \\
(\boldsymbol{\%})\end{array}$ & $\begin{array}{c}\mathbf{R}_{\mathbf{p}} \\
(\mathbf{\Omega . c m})\end{array}$ & $\begin{array}{c}\boldsymbol{\eta} \\
(\boldsymbol{\%})\end{array}$ \\
\hline $\mathrm{E}_{0}$ & 22 & - & 218.6 & - \\
\hline $\mathrm{E}_{1}$ & 1.98 & 91 & 3519 & 94 \\
\hline $\mathrm{E}_{2}$ & 0.80 & 96 & 29346 & 99 \\
\hline
\end{tabular}


That behavior is correlated with a very low barrier effect caused by the existence of pores and defects in the coating, and/or by the poor adhesion between the coating and the metal substrate. The absence of adhesion of the non-pigmented coatings is suggested by the obtained low charge transfer resistance and the rapid formation of bubbles over the entire coating surface, where the significant corrosion occurs [29]. On this basis, the larger semi-circle in the $\mathrm{E}_{2}$ coating system Nyquist diagram indicates a better corrosion resistance (see Table 3), which is explained by the presence of a natural phosphate responsible for the higher corrosion resistance.

\section{Conclusion}

After synthesizing the nanofunctional polyepoxide prepolymer nanoglycidyl trihydrazine 4,4,4-tripropoxy of ethylene tribisphenol A, we applied it for E24 steel protection by a coating process, which is derived from the formulations based on a standard epoxy prepolymer, methylene dianiline and natural phosphate used as a load. The obtained electrochemical results, namely the potentiodynamic polarization curves and those of the electrochemical impedance spectroscopy, are quite in reasonable agreement, and confirm that the natural phosphate presented in the formulated $\mathrm{E}_{2}$ matrix (NGTHTPETBA/MDA/PN) has a strong protective effect. Finally, the Bode and the phase angle diagrams confirm the electrochemical impedance spectroscopy results. In perspective we will approach the theoretical modeling study by using the DFT and the PM6 methods, in order to confirm the adhesion sites onto the metal support.

\section{References}

1. Fetouaki S, Toufik M, Meghraoui H, et al. Phys Chem New J. 2006;27:131140.

2. Hsissou R, El Rhayam Y, Elharfi A. Int J Innov Appl Stu. 2014;7:674-682.

3. Bekhta A, Lambarki El Allioui T, Bouih A, et al. Int J Innov Appl Stu. 2014;7:1057-1070.

4. El Hilal B, Elharfi A. Int J Innov Appl Stu. 2014;7:729-735.

5. Hsissou R, Dagdag O, El Harfi A. Mor J Chem. 2015;3:791-797.

6. Hsissou R, El Harfi A. Mor J Chem. 2016;4:315-323.

7. Hsissou R, Bekhta A, El Harfi A. J Mater Environ Sci. 2017;8:603-610.

8. Grich M, Meghraoui H, Ziraoui R, et al. Ann Chim Sci Mat. 2011;36:1-10.

9. El Azzaoui J, El-Aouni N, Bekhta A, et al. Mor. J. Chem. 2015;3:338-345.

10. Hsissou R, El Harfi A. Int J Phar Tech Res. 2017;10:Accepted.

11. Dagdag O, El Gouri M, Galai M, et al. Der Pharm Chem. 2015;7:114-122.

12. Dagdag O, Galai M, Ebn Touhami M, et al. J Mater Environ Sci. 2016;7:3454-3464.

13. Nakamura Y, Yamaguchi M, Okubo M, et al. J Appl Polym Sci. 1992;45:1281-1289.

14. Rosales B, Vera R, Morien G. Corros Sci. 1999;41:625-651.

15. Cantor A, Bushman J, Glodoski M, et al. Mater Performance. 2006;45:38-41. 
16. Antonijevic MM, Petrovic MB. Int J Electrochem Sci. 2008;3:1-28.

17. Petitjean J, Aeiyach S, Lacroix JC, et al. J Electroanal Chem. 1999;478:92100.

18. Panah NB, Danaee I. Prog Org Coat. 2010;68:214-218.

19. Ferreira CA, Aeiyach S, Delamar M, et al. Chem Interfacial Electrochem. 1990;284:351-369.

20. Hsissou R, Bekhta A, El Hilal B, et al. Int J Sci Eng Res. 2017;8:1181-1188.

21. Hsissou R, Rafik M, Hegazi SE, et al. Arab J Chem Envir Res. 2016;3:35-50.

22. Li W, Hu L, Zhang S, et al. Corros Sci. 2011;53:735-745.

23. Hammouti B, Dafali A, Touzani R, et al. J Saudi Chem Soc. 2012;16:413418.

24. Zubielewicz M, Gnot W. Prog Org Coat. 2004;49:358-371.

25. Rossenbeck B, Ebbinghaus P, Stratmann M, et al. Corros Sci. 2006;48:3703.

26. Liu S, Zhong Y, Jiang R, et al. Corros Sci. 2011;53:746.

27. Ananda Kumar S, Balakrishnan T, Alagar M, et al. Prog Org Coat. 2006;55:207.

28. Benali O, Larabi L, Traisnel M, et al. Appl Surf Sci. 2007;253:6130-6139.

29. Guillaumin V, Landolt D. Corros Sci. 2002;44:179. 